\title{
REQUERIMIENTO DE ENERGÍA Y PROTEÍNA PARA PATOS PEKÍN (Anas platyrhynchos) EN LAS FASES DE CRECIMIENTO Y ACABADO
}

\author{
Magdalena Herrera Gallo ${ }^{1,2}$ y Nelson Duchi Duchi ${ }^{3}$ \\ ${ }^{1}$ Unidad de Investigación Científica y Tecnológica, Universidad Técnica Estatal de Quevedo, $\mathrm{km} 1$ 1/2 vía Quevedo - \\ Santo Domingo de los Tsáchilas, C. P. 73. Quevedo, Los Ríos, Ecuador. \\ ${ }^{2}$ Facultad de Ciencias Pecuarias, Universidad Técnica Estatal de Quevedo, $\mathrm{km} 7$ vía a El Empalme \\ Mocache, Los Ríos, Ecuador \\ ${ }^{3}$ Facultad de Ciencias Pecuarias. Escuela Superior Politécnica del Chimborazo.
} Riobamba - Chimborazo - Ecuador.

Resumen

$\mathrm{C}$ on el fin de conocer los requerimientos de energía y proteína del pato Pekín (Anas platyrhinchos) en crecimiento y acabado, se utilizaron dietas isoenergéticas e isoproteicas, cinco tratamientos con cuatro repeticiones para la energía y de igual forma para la proteína. Las variables estudiadas fueron: consumo de alimento, ganancia de peso, conversión alimenticia y rendimiento a la canal. En la fase de cría se registraron diferencias significativas en las variables ganancia de peso y conversión alimenticia, no así en el consumo de alimento. No obstante, se observó que se lograron los mejores valores del consumo de alimento, ganancia de peso y conversión alimenticia, con $3010 \mathrm{kcal}^{\mathrm{de}} \mathrm{EM} \mathrm{kg}$ y $22 \% \mathrm{~PB}$ (T3), en base a la energía y con $2975 \mathrm{kcal}^{\mathrm{de}} \mathrm{EM} \mathrm{kg} \mathrm{kg}^{-1}$ y $19 \%$ de PB (T2) en base a la proteína, respectivamente. En la fase de acabado, no se registraron diferencias significativas entre los tratamientos, sin embargo. en base a la energía, el tratamiento con 2833 kcal de EM kg-1 y 18\% PB (T2) registró las mejores respuestas en las variables productivas así como en base a la proteína, la dieta con $2991 \mathrm{kcal}^{\mathrm{de}} \mathrm{EM} \mathrm{kg}^{-1}$ y 18 PB (T5).

Palabras claves: isoenergéticas, isoproteícas, crecimiento, acabado, kcal EM kg-1
$\mathrm{W}$ ith the purpose of getting to know the energy and protein required by the Pekin duck (Anas platyrhinchos) in growth and finishing phases, isoenergetic and isoproteic diets were used, five treatments with four repetitions for the energy and the equal form for the protein. The variables studied were: consumption of food, gain of weight, nutritious conversion and yield to the channel. In the growing phase significant differences in the variable gain of weight and nutritious conversion was registered. It didn't seize in the food consumption. Although, it was observed that the best consumption of food, gain of weight and nutritious conversion, were achieved, with $3010 \mathrm{kcal} \mathrm{EM} \mathrm{kg}^{-1}$ and 22\% PB (T3), based on the energy and with $2975 \mathrm{kcal} \mathrm{EM} \mathrm{kg}^{-1}$ and $19 \%$ PB based on the protein, respectively. In the finishing phase, significant differences among the treatments were not registered, however, based on the energy, the treatment with $2833 \mathrm{kcal} \mathrm{EM} \mathrm{kg}^{-1}$ and 18\% PB (T2) registered the best answers in the productive variables as well as the diet with $2991 \mathrm{kcal} \mathrm{EM} \mathrm{kg}^{-1}$ and 18 PB (T5) based on the protein.

Key words: isoenergéticas, isoproteícas, growth, finish, kcal EM kg-1

\section{INTRODUCCIÓN}

$\mathrm{L}$ os patos domésticos tienen gran relevancia como fuente de alimento en Asia, Europa, África y América Latina (SICA 2002). En Ecuador la producción tradicional (pollos y ponedoras) se encuentra en manos de las empresas avícolas con gran poder económico; el avicultor pequeño por obvias razones, no puede competir frente a ellas por lo que está obligado a desaparecer del mercado, creando un grave problema social, por lo que la cría del pato Pekín es una excelente alternativa para mejorar los índices socioeconómicos de estos productores.

En Ecuador, Quevedo, el pato no se lo ha explotado comercialmente, se lo ha criado para consumo familiar, como adorno de estanques, utilizando poca o casi nada de tecnología, con aves de baja genética, enmarcadas como "producción casera de tras patio" y que por falta de difusión se desconoce las bondades que tiene su explotación así como la exquisitez de su carne. Bajo estos antecedentes se planteó esta investigación para co-

Recibido: Junio, 2008. Aceptado: Enero, 2009.

Publicado como ARTÍCULO en Ciencia y Tecnología 3: 7-13. 2009. nocer los requerimientos nutritivos de esta ave criada en esta zona del trópico húmedo (bh-T) que pudieran servir de referente permitiendo al pequeño avicultor tener otra alternativa de producción que le servirá para mejorar sus ingresos económicos familiares.

\section{Materiales y Métodos}

T a investigación se la realizó en el plantel avícola, LFinca Experimental "La María" de la Universidad Técnica Estatal de Quevedo, localizada en el km 7 de la vía Quevedo - El Empalme, cantón Mocache, provincia de Los Ríos, ubicada a una altura de $73 \mathrm{msnm}$, con precipitación y temperatura promedio de $1326 \mathrm{~mm}$ anuales y $24.70{ }^{\circ} \mathrm{C}$, respectivamente.

En base a la energía, fase de crecimiento, se utilizaron dietas isoproteicas (22\% PB) con: 2853, 2931, 3010,3089 y $3168 \mathrm{kcal}^{\mathrm{de}} \mathrm{EM} \mathrm{kg}^{-1}$ y en acabado, dietas isoproteícas (18\% PB) con: 2733, 2833, 2933, 3033 y $3134 \mathrm{kcal}^{\mathrm{de}} \mathrm{EM} \mathrm{kg}^{-1}$. 
En base a la proteína, fase de crecimiento, se evaluaron dietas isoenergéticas (2975 kcal de EM kg-1) con: 18, 19, 20, 21 y 22\% PB y en fase de acabado, dietas isoenergéticas (2991 kcal de EM kg-1) con: 14, 15, 16,17 y $18 \%$ PB. El diseño corresponde a un completamente al azar con cinco tratamientos y cuatro repeticiones, tanto en proteína como energía, en fases de crecimiento y acabado. Los datos fueron analizados mediante el procedimiento SAS (2000). Cuando hubo diferencias estadísticas se realizaron comparaciones de las medias mediante la prueba de Tukey al $0.05 \%$ de probabilidad. Las variables de repuesta fueron: consumo de alimento, ganancia de peso, conversión alimenticia y rendimiento a la canal.

Se realizaron análisis bromatológico a cada dieta por fase con la finalidad de conocer su composición química mediante la técnica descrita por la A.O.A.C. (1990).

Cuadro 1. Dietas con diferentes niveles de energía en la fase de crecimiento (1-4 semanas) para patos Pekín.

\begin{tabular}{lrrrrr}
\hline \multicolumn{1}{c}{ Ingredientes } & 22 \% PB & 22 \% PB & 22 \% PB & 22 \% PB & 22 \% PB \\
& 2853 EM & 2931 EM & 3010 EM & 3089 EM & 3168 EM \\
\hline Maíz & 37.58 & 36.58 & 50.45 & 53.42 & 51.42 \\
Polvillo/arroz & 0.90 & 1.90 & 11.00 & 9.58 & 9.56 \\
Torta/soya & 23.00 & 20.00 & 23.00 & 21.20 & 23.20 \\
H/pescado & 10.80 & 13.80 & 9.50 & 7.90 & 9.90 \\
Aceite vegetal & 0.00 & 4.00 & 0.00 & 6.00 & 4.00 \\
Arrocillo & 24.97 & 20.97 & 3.79 & 0.00 & 0.00 \\
Alfalfa & 0.00 & 0.00 & 0.10 & 0.00 & 0.00 \\
Conchilla & 2.12 & 2.12 & 1.50 & 1.23 & 1.25 \\
H/hueso & 0.01 & 0.01 & 0.01 & 0.01 & 0.01 \\
M. Sanitìn & 0.05 & 0.05 & 0.05 & 0.05 & 0.05 \\
Sal & 0.09 & 0.09 & 0.10 & 0.11 & 0.11 \\
Metionina & 0.20 & 0.20 & 0.20 & 0.23 & 0.23 \\
Premix & 0.25 & 0.25 & 0.25 & 0.25 & 0.25 \\
Antioxidante & 0.01 & 0.01 & 0.01 & 0.01 & 0.01 \\
Total & 100.00 & 100.00 & 100.00 & 100.00 & 100.00 \\
*Costo $\$ / \mathrm{kg}$ & 0.32 & 0.30 & 0.29 & 0.29 & 0.30 \\
*Costo $\$ / 100 \mathrm{~kg}$ & 31.88 & 30.00 & 29.10 & 29.50 & 30.91 \\
\hline
\end{tabular}

Cuadro 2. Análisis calculado de las dietas con diferentes niveles de energía en la fase de crecimiento (1-4 semanas) para patos Pekín.

\begin{tabular}{|c|c|c|c|c|c|}
\hline Nutrientes & $\begin{array}{c}22 \% \text { PB } \\
2853 \text { E/M }\end{array}$ & $\begin{array}{c}22 \% \text { PB } \\
2953 \text { E/M }\end{array}$ & $\begin{array}{c}22 \% \text { PB } \\
3010 \text { E/M }\end{array}$ & $\begin{array}{l}22 \% \text { PB } \\
3089 \text { E/M }\end{array}$ & $\begin{array}{l}22 \% \text { PB } \\
3168 \mathrm{E} / \mathrm{M} \\
\end{array}$ \\
\hline Fibra (\%) & 3.90 & 3.80 & 3.70 & 3.65 & 3.60 \\
\hline Calcio (\%) & 0.80 & 0.80 & 0.79 & 0.74 & 0.74 \\
\hline Fósforo (\%) & 0.40 & 0.40 & 0.39 & 0.39 & 0.39 \\
\hline Arginina (\%) & 1.26 & 1.23 & 1.20 & 1.10 & 1.20 \\
\hline Lisina (\%) & 1.22 & 1.20 & 1.20 & 1.19 & 1.19 \\
\hline $\mathrm{M}+\mathrm{Cis}(\%)$ & 0.45 & 0.45 & 0.45 & 45.00 & 0.44 \\
\hline
\end{tabular}

Fuente: Laboratorio bromatológico Escuela Superior Politécnica del Chimborazo (ESPOCH. 2005) 
Cuadro 3. Dietas con diferentes niveles de energía en la fase de acabado (5-8 semanas) para patos Pekín.

\begin{tabular}{|c|c|c|c|c|c|}
\hline Ingredientes & $\begin{array}{l}18 \% \text { PB } \\
2733 \text { EM }\end{array}$ & $\begin{array}{l}18 \% \text { PB } \\
2833 \mathrm{EM} \\
\end{array}$ & $\begin{array}{c}18 \% . \text { PB } \\
2933 \text { EM }\end{array}$ & $\begin{array}{l}18 \% \text { PB } \\
3033 \text { EM } \\
\end{array}$ & $\begin{array}{l}18 \% \text { PB } \\
3134 \text { EM }\end{array}$ \\
\hline $\mathrm{A} /$ trigo & 4.75 & 5.49 & 7.49 & 2.00 & 1.50 \\
\hline Polvillo/arroz & 12.00 & 14.00 & 16.00 & 12.00 & 12.10 \\
\hline Torta/soya & 18.00 & 18.00 & 15.00 & 18.00 & 18.00 \\
\hline $\mathrm{H} /$ pescado & 7.00 & 9.00 & 9.00 & 7.00 & 8.00 \\
\hline Aceite vegetal & 0.00 & 0.00 & 1.00 & 1.00 & 1.70 \\
\hline Arrocillo & 18.00 & 16.00 & 7.00 & 10.07 & 2.00 \\
\hline Conchilla & 2.00 & 2.00 & 2.00 & 1.70 & 1.70 \\
\hline H/hueso & 0.00 & 0.00 & 0.00 & 0.01 & 0.01 \\
\hline M. Sanitìn & 0.05 & 0.05 & 0.05 & 0.05 & 0.05 \\
\hline Sal & 0.09 & 0.09 & 0.09 & 0.09 & 0.09 \\
\hline Metionina & 0.11 & 0.11 & 0.11 & 0.15 & 0.10 \\
\hline Premix & 0.25 & 0.25 & 0.25 & 0.25 & 0.25 \\
\hline Antioxidante & 0.01 & 0.01 & 0.01 & 0.01 & 0.01 \\
\hline Total & 100.00 & 100.00 & 100.00 & 100.00 & 100.00 \\
\hline$*$ Costo $\$ / \mathrm{kg}$ & 0.28 & 0.29 & 0.30 & 0.30 & 0.30 \\
\hline *Costo $\$ / 100 \mathrm{~kg}$ & 28.16 & 29.20 & 30.10 & 30.50 & 30.89 \\
\hline
\end{tabular}

Cuadro 4. Análisis calculado de las dietas con diferentes niveles de energía en la fase de acabado (5-8 semanas) para patos Pekín.

\begin{tabular}{|c|c|c|c|c|c|}
\hline Nutrientes & $\begin{array}{l}18 \% \text { PB } \\
2733 \text { EM }\end{array}$ & $\begin{array}{l}18 \% \text { PB } \\
2833 \text { EM }\end{array}$ & $\begin{array}{l}18 \% \text { PB } \\
2933 \text { EM }\end{array}$ & $\begin{array}{l}18 \% \text { PB } \\
3033 \text { EM }\end{array}$ & $\begin{array}{l}18 \% \text { PB } \\
3134 \mathrm{EM}\end{array}$ \\
\hline Fibra (\%) & 4.10 & 4.00 & 3.95 & 3.80 & 3.63 \\
\hline Calcio (\%) & 0.80 & 0.80 & 0.80 & 0.81 & 0.78 \\
\hline Fósforo (\%) & 0.40 & 39.00 & 0.39 & 0.39 & 0.39 \\
\hline Arginina (\%) & 1.06 & 1.04 & 1.00 & 1.03 & 1.04 \\
\hline Lisina (\%) & 1.20 & 1.20 & 1.20 & 1.18 & 1.18 \\
\hline $\mathrm{M}+\mathrm{Cis}(\%)$ & 0.42 & 0.42 & 0.42 & 41.00 & 0.41 \\
\hline
\end{tabular}

Fuente: Laboratorio bromatológico Escuela Superior Politécnica del Chimborazo (ESPOCH. 2005) 
Cuadro 5. Dietas con diferentes niveles de proteína en la fase de crecimiento (1-4 semanas) para patos Pekín

\begin{tabular}{|c|c|c|c|c|c|}
\hline Ingredientes & $\begin{array}{l}18 \% \text { PB } \\
2975 \text { EM }\end{array}$ & $\begin{array}{l}19 \% \text { PB } \\
2975 \text { EM }\end{array}$ & $\begin{array}{l}20 \% \text { PB } \\
2975 \text { EM }\end{array}$ & $\begin{array}{l}21 \% \text { PB } \\
2975 \text { EM }\end{array}$ & $\begin{array}{l}22 \% \text { PB } \\
2975 \text { EM }\end{array}$ \\
\hline Aceite vegetal & 2.10 & 2.30 & 2.70 & 3.60 & 3.80 \\
\hline Polvillo/arroz & 12.50 & 11.20 & 10.00 & 9.70 & 8.60 \\
\hline Torta/soya & 24.60 & 27.50 & 30.40 & 33.90 & 36.70 \\
\hline $\mathrm{H} / \mathrm{hueso}$ & 2.00 & 2.00 & 2.10 & 2.10 & 2.10 \\
\hline Premix & 0.50 & 0.50 & 0.50 & 0.50 & 0.50 \\
\hline Sal & 0.50 & 0.50 & 0.50 & 0.50 & 0.50 \\
\hline Total & 100.00 & 100.00 & 100.00 & 100.00 & 100.00 \\
\hline${ }^{*}$ Costo $\$ / \mathrm{kg}$ & 0.22 & 0.23 & 0.23 & 0.24 & 0.25 \\
\hline${ }^{*}$ Costo $\$ / 100 \mathrm{~kg}$ & 22.15 & 22.68 & 23.31 & 24.14 & 24.65 \\
\hline
\end{tabular}

Cuadro 6. Análisis calculado de las dietas con diferentes niveles de proteína en la fase de crecimiento (1-4 semanas) para patos Pekín

\begin{tabular}{lccccc}
\hline Nutrientes & $\begin{array}{r}\mathbf{1 8} \% \text { prot. } \\
\text { 2975 E/M }\end{array}$ & $\begin{array}{r}\mathbf{1 9} \% \text { prot. } \\
\mathbf{2 9 7 5} \text { E/M }\end{array}$ & $\begin{array}{r}\mathbf{2 0} \% \text { prot. } \\
\mathbf{2 9 7 5} \text { E/M }\end{array}$ & $\begin{array}{r}\mathbf{2 1} \text { \% prot. } \\
\mathbf{2 9 7 5} \text { E/M }\end{array}$ & $\begin{array}{c}\mathbf{2 2} \% \text { prot. } \\
\mathbf{2 9 7 5} \text { E/M }\end{array}$ \\
\hline Fibra (\%) & 3.90 & 4.00 & 4.10 & 4.20 & 4.30 \\
Calcio (\%) & 0.80 & 0.80 & 0.80 & 0.80 & 0.80 \\
Fósforo (\%) & 0.39 & 0.39 & 0.39 & 0.39 & 0.39 \\
Arginina (\%) & 0.96 & 1.03 & 1.10 & 1.18 & 1.25 \\
Lisina (\%) & 1.20 & 1.20 & 1.20 & 1.20 & 1.20 \\
M + Cis (\%) & 0.42 & 0.42 & 0.42 & 0.42 & 0.42 \\
\hline
\end{tabular}

Fuente: Laboratorio bromatológico Escuela Superior Politécnica del Chimborazo (ESPOCH. 2005) 
Cuadro 7. Dietas con diferentes niveles de proteína en la fase de acabado (5-8 semanas) para patos Pekín.

\begin{tabular}{|c|c|c|c|c|c|}
\hline Ingredientes & $\begin{array}{l}14 \% \text { PB } \\
2991 \text { EM }\end{array}$ & $\begin{array}{l}15 \% \text { PB } \\
2991 \text { EM }\end{array}$ & $\begin{array}{l}16 \% \text { PB } \\
2991 \text { EM }\end{array}$ & $\begin{array}{l}17 \% \text { PB } \\
2991 \text { ЕM }\end{array}$ & $\begin{array}{l}18 \% \text { PB } \\
2991 \text { EM }\end{array}$ \\
\hline Maíz & 63.70 & 61.70 & 59.10 & 56.00 & 57.80 \\
\hline Aceite vegetal & 1.90 & 2.20 & 2.10 & 2.30 & 2.10 \\
\hline Polvillo/arroz & 17.00 & 16.00 & 13.40 & 11.20 & 12.50 \\
\hline Torta/soya & 14.20 & 17.10 & 22.40 & 27.50 & 24.60 \\
\hline $\mathrm{H} /$ hueso & 2.10 & 2.10 & 2.00 & 2.00 & 2.00 \\
\hline Premix & 0.50 & 0.50 & 0.50 & 0.50 & 0.50 \\
\hline Sal & 0.50 & 0.50 & 0.50 & 0.50 & 0.50 \\
\hline Total & 100.00 & 100.00 & 100.00 & 100.00 & 100.00 \\
\hline Costo $\mathrm{kg}$ & 0.20 & 0.21 & 0.22 & 0.23 & 0.22 \\
\hline Costo $100 \mathrm{~kg}$ & 20.41 & 21.01 & 21.80 & 24.68 & 22.15 \\
\hline
\end{tabular}

Cuadro 8. Análisis calculado de las dietas con diferentes niveles de proteína en la fase de acabado (5-8 semanas) para patos Pekín

\begin{tabular}{|c|c|c|c|c|c|}
\hline Nutrientes & $\begin{array}{c}14 \% \text { prot. } \\
2991 \text { E/M }\end{array}$ & $\begin{array}{l}15 \% \text { prot. } \\
2991 \mathrm{E} / \mathrm{M}\end{array}$ & $\begin{array}{c}16 \% \text { prot. } \\
2991 \mathrm{E} / \mathrm{M}\end{array}$ & $\begin{array}{c}17 \% \text { prot. } \\
2991 \mathrm{E} / \mathrm{M}\end{array}$ & $\begin{array}{l}18 \% \text { prot. } \\
2991 \text { E/M }\end{array}$ \\
\hline Fibra $(\%)$ & 3.50 & 3.63 & 3.82 & 4.90 & 4.10 \\
\hline Calcio (\%) & 0.80 & 0.80 & 0.80 & 0.80 & 0.80 \\
\hline Fósforo (\%) & 0.40 & 0.40 & 0.40 & 0.40 & 0.40 \\
\hline Arginina (\%) & 0.72 & 0.79 & 0.91 & 1.03 & 1.04 \\
\hline Lisina (\%) & 1.20 & 1.20 & 1.20 & 1.20 & 1.20 \\
\hline $\mathrm{M}+\mathrm{C}$ is $(\%)$ & 0.43 & 0.45 & 0.50 & 0.54 & 0.54 \\
\hline
\end{tabular}

Fuente: Laboratorio bromatológico Escuela Superior Politécnica del Chimborazo (ESPOCH. 2005)

\section{Resultados y Discusión}

$\mathrm{L}_{\mathrm{p}}^{\mathrm{o}}$ os resultados de la fase de crecimiento en energía y proteína de los tratamientos en estudio, se presentan en los Cuadros 9 y 10.

En fase de crecimiento, con base en la energía y a la proteína, se registraron diferencias significativas en las variables: ganancia de peso y conversión alimenticia, lo contrario ocurrió con la variable, consumo de alimento. Sin embargo, el tratamiento con $22 \%$ de PB y 3010 kcal de EM kg ${ }^{-1}$ MS (T3) logró las mejores respuestas en las variables: ganancia de peso y conversión alimenticia, no obstante, el mayor consumo lo presentó el tratamiento con 22\% PB y 3168 kcal de $\mathrm{EM} \mathrm{kg}^{-1}$ MS (T5) con base en la energía y con base en la proteína, el tratamiento con $19 \%$ de PB y $2975 \mathrm{kcal}$ de EM $\mathrm{kg}^{-1}$ MS (T2), alcanzó los mejores resultados frente a los demás tratamientos, concordando con lo encontrado por Siregar, Farrell y Dean 1980ª citados por Lázaro et al., 2004, quiénes manifiestan que el pato Pekín en sus primeros estadios requiere de alta concentración energética y proteica para maximizar su consumo, además que el pato ajusta el requerimiento calórico mejor que el pollo, ya que éste tiene mayor tendencia a sobre consumir dietas muy concentradas; observaron también que en el periodo de crecimiento, el contenido de proteína bruta debe estar entre el 18 a 19\% PB para que el pato aumente su consumo, Jeroch y Flachowsky (1978); Dean 1972, citado por Lázaro et al., 2004, recomiendan utilizar niveles de proteína del 22\% PB en esta fase, lo que se ajusta a los resultados encontrados en esta investigación.

En la fase de acabado, con base en la energía y proteína no se encontraron diferencias significativas entre tratamientos, no obstante, la dieta con $18 \%$ de PB y $2833 \mathrm{kcal} \mathrm{de} \mathrm{EM} \mathrm{kg}^{-1} \mathrm{MS}$ (T2) en base a la energía y el tratamiento con $18 \%$ de PB y $2991 \mathrm{kcal} \mathrm{de} \mathrm{EM} \mathrm{kg-1}$ MS (T5), con base en la proteína, optimizaron los rendimientos productivos de las variables estudiadas (Cuadro 11 y 12). Los resultados demuestran que el pato tuvo preferencia por dietas con $18 \%$ de proteína, mostrando indiferencia por la concentración energética de las 
mismas, lo que coincide con los resultados encontrados; Auvergne et al. y Mercia 1988, citado por Lázaro et al., 2004, señalan que el pato Pekín aprovecha bien dietas que contenga $22 \%$ PB las dos primeras semanas de vida y luego, aquellas con niveles entre 15 a $18 \%$ PB, sin afectar a la ganancia de peso, conversión alimenticia y rendimiento a la canal, por lo que se ratifica que el pato muestra preferencia por dietas donde se mantiene una relación equilibrada entre la energía y proteína (158170). Scott et al. 1991, citado por Lázaro et al., 2004 y Leclercq et al. 1976 , citado por Lázaro et al., 2004.

Cuadro 9. Consumo de alimento, ganancia de peso y conversión alimenticia de la fase de crecimiento con base en la energía, del pato Pekín. Finca "La María" UTEQ, 2005

\begin{tabular}{|c|c|c|c|c|c|c|}
\hline Variables & T1* & T2 & T3 & $\mathrm{T4}$ & T5 & Probab \\
\hline Consumo de alimento (g) & $2334.50 \mathrm{a}$ & $2490.50 \mathrm{a}$ & $2391.00 \mathrm{a}$ & $2514.30 \mathrm{a}$ & $2651.90 \mathrm{a}$ & 0.156 \\
\hline Ganancia de peso (g) & $1008.75 \mathrm{c}$ & $1149.50 \mathrm{~b}$ & $1263.00 \mathrm{a}$ & $1208.50 \mathrm{a}$ & $1115.75 \mathrm{~b}$ & 0.000 \\
\hline Conversión alimenticia & $2.31 \mathrm{a}$ & $2.16 \mathrm{ab}$ & $1.89 \mathrm{~b}$ & $2.08 \mathrm{ab}$ & 2.38 & 0.023 \\
\hline
\end{tabular}

*Medias con letras iguales no presentan diferencias estadísticas según la Prueba de Tukey $(\mathrm{P} \leq 0.05)$

$\mathrm{T} 1=22 \%$ Proteína - $2853 \mathrm{kcal}$ de EM MS kg-1 T4 = 22\% PB - 3089 Kcal de EM MS kg-1

$\mathrm{T} 2=22 \%$ Proteína $-2931 \mathrm{kcal}$ de EM MS kg-1 $\mathrm{T} 5=22 \%$ PB $-3168 \mathrm{kcal}$ de EM MS kg-1

$\mathrm{T} 3=22 \%$ PB $-3010 \mathrm{Kcal}$ de EM MS kg-1

Cuadro 10. Consumo de alimento, ganancia de peso y conversión alimenticia en la fase de crecimiento con base en la proteína del pato Pekín. Finca "La María" UTEQ. 2005

\begin{tabular}{lccccccc}
\hline \multicolumn{1}{c}{ Variables } & T1* & T2 & T3 & T4 & T5 & Prob \\
\hline Consumo de alimento $(\mathrm{g})$ & $2393.0 \mathrm{a}$ & $2431.85 \mathrm{a}$ & $2422.33 \mathrm{a}$ & $2399.5 \mathrm{a}$ & $2422 . .0 \mathrm{a}$ & 0.688 \\
Ganancia de peso $(\mathrm{g})$ & $1130.5 \mathrm{~b}$ & $1281.25 \mathrm{a}$ & $1198.95 \mathrm{ab}$ & $1262.7 \mathrm{a}$ & $1272.25 \mathrm{a}$ & 0.01 \\
Conversión alimenticia & $2.2 \mathrm{a}$ & $1.90 \mathrm{~b}$ & $2.02 \mathrm{ab}$ & $1.98 \mathrm{~b}$ & 2.41 & $\mathrm{~b}$ & 0.003 \\
\hline
\end{tabular}

*Medias con letras iguales no presentan diferencias estadísticas según la Prueba de Tukey $(\mathrm{P} \leq 0.05)$

$\mathrm{T} 1=18 \%$ Proteína - $2975 \mathrm{kcal}$ de EM MS kg-1 $\quad \mathrm{T} 4=21 \%$ Proteína - $2975 \mathrm{Kcal}^{\mathrm{N}}$ de EM MS kg-1

$\mathrm{T} 2=19 \%$ Proteína - $2975 \mathrm{kcal}$ de EM MS kg-1 T5 = 22\% Proteína - 2975 kcal de EM MS kg-1

$\mathrm{T} 3=20 \%$ Proteína $-2975 \mathrm{Kcal}$ de EM MS kg-1

Cuadro 11. Consumo de alimento, ganancia de peso y conversión alimenticia en la fase de acabado con base en la energía del pato Pekín. Finca "La María” UTEQ. 2005

\begin{tabular}{lcccccc}
\hline \multicolumn{1}{c}{ Variables } & T1* & T2 & T3 & T4 & T5 & Prob \\
\hline Consumo de alimento $(\mathrm{g})$ & $5253.6 \mathrm{a}$ & $5274.00 \mathrm{a}$ & $5183.50 \mathrm{a}$ & $4895.80 \mathrm{a}$ & $5181.80 \mathrm{a}$ & 0.516 \\
Ganancia de peso $(\mathrm{g})$ & $1845.5 \mathrm{a}$ & $1894.00 \mathrm{a}$ & $1848.25 \mathrm{a}$ & $1759.50 \mathrm{a}$ & $1807.00 \mathrm{a}$ & 0.363 \\
Conversión alimenticia & $2.86 \mathrm{a}$ & $2.77 \mathrm{a}$ & $2.80 \mathrm{a}$ & $2.78 \mathrm{a}$ & $2.87 \mathrm{a}$ & 0.396 \\
Rendimiento canal $(\%)$ & $62.02 \mathrm{a}$ & $64.04 \mathrm{a}$ & $64.02 \mathrm{a}$ & $61.06 \mathrm{a}$ & $62.27 \mathrm{a}$ & 0.739 \\
\hline
\end{tabular}

\footnotetext{
* Medias con letras iguales no presentan diferencias estadísticas según la Prueba de Tukey $(\mathrm{P} \leq 0.05)$

$\mathrm{T} 1=18 \%$ Proteína $-2733 \mathrm{kcal}$ de EM MS kg-1 T4 = 18\% Proteína - 3033 Kcal de EM MS kg-1

$\mathrm{T} 2=18 \%$ Proteína $-2833 \mathrm{kcal}$ de EM MS kg-1 $\mathrm{T} 5=18 \%$ Proteína -3134 kcal de EM MS kg-1

$\mathrm{T} 3=18 \%$ Proteína $-2933 \mathrm{kcal}$ de EM MS kg-1
} 
Cuadro 12. Consumo de alimento, ganancia de peso y conversión alimenticia en la fase de acabado con base en la proteína del pato Pekín. Finca "La María". UTEQ. 2005

\begin{tabular}{lcccccc}
\hline \multicolumn{1}{c}{ Variables } & T1* & T2 & T3 & T4 & T5 & Prob \\
\hline Consumo de alimento $(\mathrm{g})$ & $5174.70 \mathrm{a}$ & $5177.20 \mathrm{a}$ & $5215.00 \mathrm{a}$ & $5115.50 \mathrm{a}$ & $5281.50 \mathrm{a}$ & 0.528 \\
Ganancia de peso $(\mathrm{g})$ & $1895.25 \mathrm{a}$ & $1870.40 \mathrm{a}$ & $1858.28 \mathrm{a}$ & $1883.38 \mathrm{a}$ & $1936.20 \mathrm{a}$ & 0.54 \\
Conversión alimenticia & $2.84 \mathrm{a}$ & $2.77 \mathrm{a}$ & $2.81 \mathrm{a}$ & $2.73 \mathrm{a}$ & $2.72 \mathrm{a}$ & 0.234 \\
Rendimiento canal $(\%)$ & $59.05 \mathrm{a}$ & $60.01 \mathrm{a}$ & $59.05 \mathrm{a}$ & $60.08 \mathrm{a}$ & $62.04 \mathrm{a}$ & 0.312 \\
\hline
\end{tabular}

*Medias con letras iguales no presentan diferencias estadísticas según la Prueba de Tukey $(\mathrm{P} \leq 0.05)$

$\mathrm{T} 1=14 \%$ Proteína $-2991 \mathrm{kcal}^{\mathrm{Ne}} \mathrm{EM} \mathrm{kg}^{-1} \mathrm{MS} \quad \mathrm{T} 4=17 \%$ Proteína $-2991 \mathrm{kcal} \mathrm{de} \mathrm{EM} \mathrm{kg}^{-1}$

$\mathrm{T} 2=15 \%$ Proteína $-2991 \mathrm{kcal}_{\mathrm{de}} \mathrm{EM} \mathrm{kg}^{-1} \mathrm{MS} \quad \mathrm{T} 5=18 \%$ Proteína $-2991 \mathrm{kcal} \mathrm{de} \mathrm{EM} \mathrm{kg}{ }^{-1}$

$\mathrm{T} 3=16 \%$ Proteína $-2991 \mathrm{kcal}^{\mathrm{de}} \mathrm{EM} \mathrm{kg}^{-1} \mathrm{MS}$

\section{COnClusiones}

T os patos Pekín mejoraron sus respuestas productivas como se indica a continuación:

En base a la energía, fase de crecimiento con $3010 \mathrm{kcal}_{\mathrm{de}} \mathrm{EM} \mathrm{kg}{ }^{-1} \mathrm{MS}$ y $22 \%$ PB.

Fase de acabado, $2833 \mathrm{kcal}^{\mathrm{de}} \mathrm{EM} \mathrm{kg}^{-1} \mathrm{MS}$ con $18 \% \mathrm{~PB}$

En base a la proteína, fase de crecimiento con $18 \%$ PB y $2991 \mathrm{kcal}^{\mathrm{E}} \mathrm{EM} \mathrm{kg} \mathrm{kS}^{-1} \mathrm{MS}$.

Fase de acabado, 19\% PB y $2975 \mathrm{kcal}$ de EM $\mathrm{kg}^{-1} \mathrm{MS}$

\section{Literatura Citada}

A.O.A.C. 1990. Oficial Methods of Analisis of A.O.A.C 15 th. Ed. Vol. Assoc of Analysis Chemest. Arlintong USA.Sas Institute Inc. 2002. SAS user`s guide: Statistics. Cary. EEUU.. SAS Institute Inc. 629 p.
SICA. 2002. III Censo Nacional Agropecuario. Servicio de Información Censo Agropecuario. Ministerio de Agricultura y Ganadería. Instituto Nacional de Estadística y Censos.

Lázaro. R; Vicente. B; Capdevila. J. 2004. Nutrición y alimentación de Avicultura Complementaria Patos. Departamento de Producción Animal. Universidad Politécnica de Madrid. XX Curso de Especialización FEDNA Jeroch, Flachowsky. G. 1978. Nutrición de aves. Patos de engorde. Zaragoza España, Acribia. Pp. 116 - 123.

SAS. 2000. SAS/STAT. User's Guide (4 ${ }^{\text {Th }}$ Ed.). SAS Institute Inc. Cary, NC 